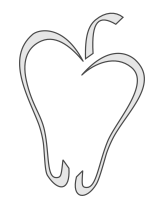

ISSN 1852-4249

\title{
Topological quantum phase transition in strongly correlated Kondo insulators in $1 \mathrm{D}$
}

\author{
Franco T. Lisandrini, ${ }^{1,2}$ Alejandro M. Lobos, ${ }^{1}$ Ariel O. Dobry, ${ }^{1,2}$ Claudio J. Gazza ${ }^{1,2 *}$
}

\begin{abstract}
We investigate, by means of a field-theory analysis combined with the density-matrix renormalization group (DMRG) method, a theoretical model for a strongly correlated quantum system in one dimension realizing a topologically-ordered Haldane phase ground state. The model consists of a spin- $1 / 2$ Heisenberg chain coupled to a tight-binding chain via two competing Kondo exchange couplings of different type: a " $s$-wave" Kondo coupling $\left(J_{K}^{s}\right)$, and a less common " $p$-wave" $\left(J_{K}^{p}\right)$ Kondo coupling. While the first coupling is the standard Kondo interaction studied in many condensed-matter systems, the latter has been recently introduced by Alexandrov and Coleman [Phys. Rev. B 90, 115147 (2014)] as a possible mechanism leading to a topological Kondo-insulating ground state in one dimension. As a result of this competition, a topological quantum phase transition (TQPT) occurs in the system for a critical value of the ratio $J_{K}^{s} / J_{K}^{p}$, separating a (Haldane-type) topological phase from a topologically trivial ground state where the system can be essentially described as a product of local singlets. We study and characterize the TQPT by means of the magnetization profile, the entanglement entropy and the full entanglement spectrum of the ground state. Our results might be relevant to understand how topologically-ordered phases of fermions emerge in strongly interacting quantum systems.
\end{abstract}

\section{Introduction}

The study of topological quantum phases of matter has become an area of great interest in present-day condensed matter physics. A topological phase is a quantum phase of matter which cannot be characterized by a local order parameter, and thus falls beyond the Landau paradigm. In particular, topological insulators (i.e., materials which are insulating in the bulk but support topologically protected gapless states at the edges) were first proposed the-

\footnotetext{
*E-mail: gazza@ifir-conicet.gov.ar
}

1 Instituto de Física Rosario (CONICET), Bv 27 de Febrero 210 bis, S2000EZP Rosario, Santa Fe, Argentina.

2 Facultad de Ciencias Exactas, Ingeniería y Agrimensura, Universidad Nacional de Rosario, Argentina. oretically for two- and three-dimensional systems with time-reversal symmetry [1-3], and soon after found in experiments on HgTe quantum wells [4] and in $\mathrm{Bi}_{1-x} \mathrm{Sb}_{x}$ [5], and $\mathrm{Bi}_{2} \mathrm{Se}_{3}$ [6], generating a lot of excitement and subsequent research. The electronic structure of a topological insulator cannot be smoothly connected to that of a trivial insulator, a fact that is mathematically expressed in the existence of a nonzero topological invariant, an integer number quantifying the non-local topological order in the ground state. A complete classification based on the dimensionality and underlying symmetries has been achieved in the form of a "periodic table" of topological insulators [7-9]. Nevertheless, this classification refers only to the gapped phases of noninteracting fermions, and leaves open the problem of characterizing and classifying strongly in- 
PAPers in Physics, vol. 8, ART. 080005 (2016) / F. T. Lisandrini et al.

teracting topological insulators. This is a very important open question in modern condensed-matter physics.

Recently, Dzero et al. [10-12] proposed a new kind of topological insulator: the topological Kondo insulator (TKI), which combines features of both non-interacting topological insulators and the well-known Kondo insulators, a special class of heavy-fermion system with an insulating gap strongly renormalized by interactions. Within a mean-field picture, TKIs can be understood as a strongly renormalized $f$-electron band lying close to the Fermi level, and hybridizing with the conduction-electron $d$-bands [13-15]. At halffilling, an insulating state appears due to the opening of a low-temperature hybridization gap at the Fermi energy induced by interactions. Due to the opposite parities of the states being hybridized, a topologically non-trivial ground state emerges, characterized by an insulating gap in the bulk and conducting Dirac states at the surface [10]. At present, TKI materials, among which samarium hexaboride $\left(\mathrm{SmB}_{6}\right)$ is the best known example, are under intense investigation both theoretically and experimentally [16-19] .

From a theoretical point of view, TKIs are interesting systems arising from the interplay between strong interactions and topology. Although the large- $N$ meanfield approach was successful in describing qualitatively heavy-fermion systems and TKIs in particular, it would be desirable to understand better how TKIs emerge. In order to shed more light into this question, in a recent work Alexandrov and Coleman proposed a onedimensional model for a topological Kondo insulator $[20]$, the " $p$-wave" Kondo-Heisenberg model $(p$ KHM). Such a model consists of a chain of spin- $1 / 2$ magnetic impurities interacting with a half-filled one-dimensional electron gas through a Kondo exchange (see Fig. 1). Using a standard mean-field description [13-15], which expresses the original interacting problem as an effectively non-interacting one, the authors mentioned above found a topologically non-trivial insulating ground state (i.e., topological class D [7-9]) which hosts magnetic states at the open ends of the chain. However, this system was studied recently using the Abelian bosonization approach combined with a perturbative renormalization group analysis, revealing an unexpected connection to the Haldane phase at low tempera- tures [21]. The Haldane phase is a paradigmatic example of a strongly interacting topological system [22-26]. The results in Ref. [21] indicate that 1D TKI systems might be much more complex and richer than expected with the naïve mean-field approach, as they are uncapable of describing the full complexity of the Haldane phase, and suggest that they must be reconsidered from the more general perspective of interacting symmetry-protected topological (SPT) phases [25-28]. More recently, two numerical studies using exact DMRG methods have confirmed that $1 \mathrm{D}$ TKIs belong to the universality class of Haldane insulators $[29,30]$. These studies have extended the regime of validity of the results in Ref. [21].

In this work, we theoretically investigate the robustness of the Haldane phase in one-dimensional topological Kondo insulators, and study the effect of local interactions that destabilize the topological phase and drive the system to a non-topolgical phase. Our goal is to characterize the system at, and near to, the topological quantum phase transition (TQPT) from the perspective of symmetryprotected topological phases, using the concepts of entanglement entropy and entanglement spectrum to detect the topologically-ordered ground states. This is a novel perspective in the context of TKIs, which might shed new light on the emergence of topological order on strongly correlated phases of fermions, and makes our work interesting from the pedagogical and conceptual points of view.

\section{Theoretical model}

We describe the system depicted in Fig. 1 with the Hamiltonian $H=H_{1}+H_{2}+H_{K}^{(s)}+H_{K}^{(p)}$, where the conduction band is represented by a $L$-site tightbinding chain

$$
H_{1}=-t \sum_{j=1, \sigma}^{L-1}\left(c_{j, \sigma}^{\dagger} c_{j+1, \sigma}+\text { H.c. }\right),
$$

with $c_{j, \sigma}^{\dagger}$, the creation operator of an electron with spin $\sigma$ at site $j$. The Hamiltonian

$$
H_{2}=J_{H} \sum_{j=1}^{L-1} \mathbf{S}_{j} \cdot \mathbf{S}_{j+1} \quad\left(J_{H}>0\right),
$$




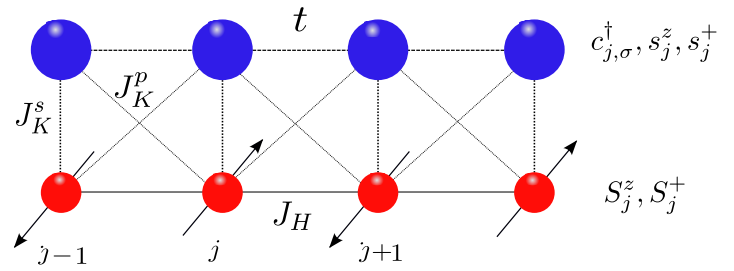

Figure 1: Sketch of the Kondo-Heisenberg model under consideration. The lower leg represents a spin-1/2 Heisenberg chain with $J_{H}>0$. The upper leg represents a half-filled one-dimensional tight binding chain interacting with the lower leg through two different Kondo exchange couplings, a "s-wave" $J_{K}^{s}$ and a " $p$-wave" $J_{K}^{p}$. We also show the fermionic and spin operators defined on each eight-dimensional "supersite" $j$ (see text).

corresponds to a spin- $1 / 2$ antiferromagnetic Heisenberg chain. The terms $H_{K}^{(s)}$ and $H_{K}^{(p)}$ describe two different types of Kondo exchange couplings between $H_{1}$ and $H_{2}$, namely

$$
\begin{aligned}
& H_{K}^{(s)}=J_{K}^{s} \sum_{j=1}^{L} \mathbf{S}_{j} \cdot \mathbf{s}_{j}, \\
& H_{K}^{(p)}=J_{K}^{p} \sum_{j=1}^{L} \mathbf{S}_{j} \cdot \boldsymbol{\pi}_{j},
\end{aligned}
$$

with $J_{K}^{a}>0(a=s, p)$. Eq. (3) describes the usual antiferromagnetic Kondo exchange coupling of a spin $\mathbf{S}_{j}$ in the Heisenberg chain to the local spin density in the fermionic chain at site $j$, defined as:

$$
\mathbf{s}_{j} \equiv \sum_{\alpha, \beta} c_{j, \alpha}^{\dagger}\left(\frac{\boldsymbol{\sigma}_{\alpha \beta}}{2}\right) c_{j, \beta},
$$

where $\boldsymbol{\sigma}_{\alpha \beta}$ is the vector of Pauli matrices. On the other hand, Eq. (4) describes a " $p$-wave" Kondo interaction, which is unusual in that it couples the spin $\mathbf{S}_{j}$ to the $p$-wave spin density in the fermionic chain at site $j$, defined as: [20]

$$
\begin{gathered}
\boldsymbol{\pi}_{j} \equiv \\
\sum_{\alpha, \beta}\left(\frac{c_{j+1, \alpha}^{\dagger}-c_{j-1, \alpha}^{\dagger}}{\sqrt{2}}\right)\left(\frac{\boldsymbol{\sigma}_{\alpha \beta}}{2}\right)\left(\frac{c_{j+1, \beta}-c_{j-1, \beta}}{\sqrt{2}}\right),
\end{gathered}
$$

where the notation $c_{0, \sigma}=c_{L+1, \sigma}=0$ is implied.

The case $J_{K}^{s}>0$ and $J_{K}^{p}=0$ corresponds to the so-called "Kondo-Heisenberg model" in 1D, which has been extensively studied in the past in connection to the stripe phase of high- $T_{c}$ superconductors [31-37]. These previous works indicate that, at half-filling, that model does not support any topological phases. On the other hand, the case $J_{K}^{s}=0$ and $J_{K}^{p}>0$ is the " $p$-wave" Kondo-Heisenberg model proposed recently in Ref. [20], and subsequently studied in Refs. [21, 29,30], where it was stablished that the ground state corresponds to the Haldane phase. In both cases, at halffilling the system develops a Mott-insulating gap in the fermionic chain due to "umklapp" processes (i.e., backscattering processes with $2 k_{F}$ momentum transfer) originated in the Kondo interactions $J_{K}^{s}$ or $J_{K}^{p}$, and at low temperatures (lower than the Mott gap), the system effectively maps onto a spin- $1 / 2$ ladder. However, the ground states of the model in both cases cannot be smoothly connected (i.e., one is topologically trivial while the other is not), and therefore we anticipate that a gap-closing topological quantum phase transition (TQPT) must occur as a function of the ratio $J_{K}^{s} / J_{K}^{p}$. Indeed, in Ref. [21], it was proposed that while in the first case the (effective) spin- $1 / 2$ ladder forms singlets along the rungs, in the second case the Kondo coupling $J_{K}^{p}$ favors the formation of triplets on the rungs, and therefore the system maps onto the Haldane spin-1 chain [38-41]. From this perspective, our toy-model Hamiltonian $H$ allows to explore a transtition from topological to non-topological Kondo insulators, and to gain a valuable insight of the TQPT.

\section{Field-theory analysis}

The purpose of this section is to provide a simple and phenomenological understanding of the competition between the two Kondo interactions $J_{K}^{s}$ and $J_{K}^{p}$, which is not evident in Eqs. (3) and (4). To that end, we introduce a field-theoretical representation of the model, valid at sufficiently low temperatures. We linearize the non-interacting spectrum $\epsilon_{k}=-2 t \cos (k a)$ in the tight-binding chain $H_{1}$ around the Fermi energy $\mu=0$, and take the continuum limit $a \rightarrow 0$, where $a$ is the lattice constant. Then, the low-energy representation of the 
PAPers in Physics, vol. 8, ART. 080005 (2016) / F. T. Lisandrini et al.

fermionic operators becomes [42-46]

$$
\frac{c_{j, \sigma}}{\sqrt{a}} \sim e^{i k_{F} x_{j}} R_{1, \sigma}\left(x_{j}\right)+e^{-i k_{F} x_{j}} L_{1, \sigma}\left(x_{j}\right),
$$

where $R_{1, \sigma}(x)$ and $L_{1, \sigma}(x)$ are right- and leftmoving fermionic field operators, which vary slowly on the scale of $a$. Using this representation, the spin densities become [47]:

$$
\begin{aligned}
\frac{\mathbf{s}_{j}}{a} \rightarrow & {\left[\mathbf{J}_{1 R}\left(x_{j}\right)+\mathbf{J}_{1 L}\left(x_{j}\right)+(-1)^{j} \mathbf{N}_{1}\left(x_{j}\right)\right], } \\
\frac{\boldsymbol{\pi}_{j}}{a} \rightarrow 2[ & {\left[\mathbf{J}_{1 R}\left(x_{j}\right)+\mathbf{J}_{1 L}\left(x_{j}\right)\right.} \\
& \left.\quad-(-1)^{j} \mathbf{N}_{1}\left(x_{j}\right)\right],
\end{aligned}
$$

where we have defined slowly-varying spin densities for the smooth spin configurations:

$$
\begin{aligned}
& \mathbf{J}_{1 R}\left(x_{j}\right)=\sum_{\alpha, \beta} R_{1, \alpha}^{\dagger}\left(x_{j}\right)\left(\frac{\boldsymbol{\sigma}_{\alpha \beta}}{2}\right) R_{1, \beta}\left(x_{j}\right), \\
& \mathbf{J}_{1 L}\left(x_{j}\right)=\sum_{\alpha, \beta} L_{1, \alpha}^{\dagger}\left(x_{j}\right)\left(\frac{\boldsymbol{\sigma}_{\alpha \beta}}{2}\right) L_{1, \beta}\left(x_{j}\right),
\end{aligned}
$$

and for the staggered spin density

$$
\mathbf{N}_{1}\left(x_{j}\right)=\sum_{\alpha, \beta} R_{1, \alpha}^{\dagger}\left(x_{j}\right)\left(\frac{\boldsymbol{\sigma}_{\alpha \beta}}{2}\right) L_{1, \beta}\left(x_{j}\right)+\text { H.c.. }
$$

Similarly, a continuum representation for the Heisenberg chain can be achieved, e.g., by fermionization of the $S=1 / 2$ spins by means of a JordanWigner transformation. At low energies, the spin densities become [38-41,47]

$$
\frac{\mathbf{S}_{j}}{a} \rightarrow \mathbf{J}_{2 R}\left(x_{j}\right)+\mathbf{J}_{2 L}\left(x_{j}\right)+(-1)^{j} \mathbf{N}_{2}\left(x_{j}\right) .
$$

We now focus on the Kondo interaction and leave the analysis of $H_{1}$ and $H_{2}$ aside, as these terms are unimportant for the qualitative understading of the basic mechanism leading to the TQPT. Keeping the most relevant (in the RG sense) terms, we can write the Kondo interaction as:

$$
\begin{aligned}
H_{K}^{(s)}+H_{K}^{(p)} & \rightarrow\left(J_{K}^{s}-2 J_{K}^{p}\right) \int d x \mathbf{N}_{1}(x) \cdot \mathbf{N}_{2}(x) \\
& (+ \text { less relevant contributions }),
\end{aligned}
$$

i.e., the Kondo interaction couples the staggered magnetization components in chains 1 and 2 . In the above expression, note that while a large $J_{K}^{s}$ favors a positive value of the effective coupling $\left(J_{K}^{s}-2 J_{K}^{p}\right)$, therefore promoting the formation of local singlets along the rungs, a large $p$-wave Kondo coupling $J_{K}^{p}$ favors an effective ferromagnetic coupling which promotes the formation of local triplets with $S=1$ (hence the connection to the $S=1$ Haldane chain). The minus sign in front of $J_{K}^{p}$ appears as a result of the $p$-wave nature of the orbitals in Eq. (6). From this qualitative analysis we can conclude that $J_{K}^{s}$ and $J_{K}^{p}$ will be competing interactions promoting different ground states, and since these grounstates cannot be adiabatically connected with each other, a TQPT must occur.

Strictly speaking, near the critical region where the bare coupling $\left(J_{K}^{s}-2 J_{K}^{p}\right)$ vanishes, the less relevant terms neglected in Eq. (14) should be taken into account. However, note that operators with conformal spin 1 (i.e., operators of the form $\left(\partial_{x} \mathbf{N}_{1}\right) \cdot \mathbf{N}_{2}$ or $\mathbf{N}_{1} \cdot\left(\partial_{x} \mathbf{N}_{2}\right)$, see for instance Ref. [48]) are not allowed by the inversion symmetry of the Hamiltonian and the $p$-wave symmetry of the orbitals in Eq. (6), which demands that $c_{j+1, \alpha}-c_{j-1, \alpha} \rightarrow-\left(c_{-(j+1), \alpha}-c_{-(j-1), \alpha}\right)$ under the change $j \rightarrow-j$, and therefore forbids the occurrence of terms proportional to $\partial_{x} \mathbf{N}_{1}$. Therefore, only the marginal operators $\mathbf{J}_{1 \nu} . \mathbf{J}_{2 \nu^{\prime}}$ (with $\nu=\{R, L\})$ and terms with conformal spin bigger than 1 are expected in the Hamiltonian. We do not expect these operators to change the physics qualitatively near the critical point, and we can ignore them for this simplified analysis. As we show below, our numerical DMRG results are in accordance with this qualitative picture.

\section{DMRG analysis}

Before presenting the numerical results, it is worth providing technical details on the implementation of the DMRG method applied to the present model. This particular Hamiltonian contains two types of terms: (a) terms involving two local operators, as in most condensed-matter models with nearestneighbor interactions, i.e., Eqs. (1)-(3), and (b) terms involving three local operators, which result from the expansion of Eq. (4). To make it easier to implement, we have found useful to define first 
PAPers in Physics, vol. 8, ART. 080005 (2016) / F. T. Lisandrini et al.

a "supersite" representation of the system, where each supersite combines a spin $\mathbf{S}_{j}$ and the fermionic site along each rung (see Fig. 1), therefore spanning a new 8-dimensional local basis. The first kind of terms could be easily handled with standard DMRG implementations where the system is represented as $L(j) \otimes \bullet \otimes \bullet \otimes R(N-j-2)$, with $L(j)$ and $R(j)$ the left and right blocks with $j$ supersites, respectively, and the two circles are the exactlyrepresented middle supersites $j+1$ and $j+2$. This comes at a price, however, since one is then forced to re-express the electron creation operator $c_{j, \sigma}^{\dagger}$, and the spin-1/2 operator $\mathbf{S}_{j}$ in this new basis in order to implement Eqs. (1) and (2). In this basis, note that the Hamiltonian $H_{K}^{(s)}$, Eq. (3), becomes an "on-site" term, which can be handled easily.

In the second type of contributions, the presence of three-operator terms in $H_{K}^{(p)}$, Eq. (4), must be properly treated in order to avoid extra truncation errors due to the tensor product of two (already truncated) operators inside each left and right blocks. Then, during each left-right DMRG sweep iteration, we save in the previous superblock configuration $L(j-1) \otimes \bullet \otimes \bullet \otimes R(N-j-3)$ the exact correlation matrices between spin and fermionic operators that involve positions $j-1$ (i.e., rightmost supersite of left block) and $j$ (first single site), which will become the two rightmost supersites of the new $L(j)$ in the next sweep iteration step. These correlation matrices are

$$
\begin{aligned}
{\left[A_{j-1, j, \sigma}^{z \dagger}\right]_{i ; i^{\prime}} } & = \\
& \rho_{i ; i 1 i 2}\left[S_{j-1}^{z}\right]_{i 1 ; i 1^{\prime}}\left[c_{j, \sigma}^{\dagger}\right]_{i 2 ; i 2^{\prime}} \rho_{i 1^{\prime} i 2^{\prime} ; i^{\prime}}^{\dagger}
\end{aligned}
$$

and

$$
\begin{aligned}
{\left[A_{j-1, j, \bar{\sigma}}^{+\dagger}\right]_{i ; i^{\prime}} } & = \\
& \rho_{i ; i 1 i 2}\left[S_{j-1}^{+}\right]_{i 1 ; i 1^{\prime}}\left[c_{j, \bar{\sigma}}^{\dagger}\right]_{i 2 ; i 2^{\prime}} \rho_{i 1^{\prime} i 2^{\prime} ; i^{\prime}}^{\dagger}
\end{aligned}
$$

where $\rho_{i ; i 1 i 2}$ is the $m \times 8 m$ reduced density matrix, with $m$ the number of states kept, and where the indices $i$ and $i 1$ run over the truncated $m$-dimensional Hilbert space while $i 2$ runs over the 8 -dimensional supersite space. In addition, we have assumed summation over repeated internal indices. Similarly, correlations between spin and fermionic operators placed in the two leftmost supersites $j+3$ and $j+4$ of $R(N-j-2)$ should also be kept during the corresponding step of the rightf-left sweep. Therefore, we now deal with a standard "two-operator" interaction, for example the term $L(j) \otimes \bullet$ of $H_{K}^{(p)}$ is

$-\frac{1}{4} J_{K}^{p} \sum_{\sigma}\left\{\left(\sigma A_{j-1, j, \sigma}^{z \dagger}+A_{j-1, j, \bar{\sigma}}^{+\dagger}\right) c_{l_{j+1}, \sigma}+\right.$ h.c. $\}$.

Finally, we mention that in our implementation we have kept up to a maximum of 800 states and we have swept 12 times, assuring truncation errors in the density matrix of the order $10^{-9}$ at worst.

We now turn to the results. We have used the tight-binding parameter $t$ as our unit of energies, and in all of our calculations we have used the values $J_{H} / t=1$ and $J_{K}^{p} / t=2$. We have studied the evolution of the ground state upon the increase of $J_{K}^{s}$ starting from the value $J_{K}^{s}=0$, where the system is in the Haldane phase. The nature of the topologically-ordered ground state and the precise detection of the TQPT are determined using, respectively: a) the analysis of the spin profile in the ground state, b) the value of the entanglement entropy and c) the analysis of the degeneracies in the full entanglement spectrum. As shown recently in seminal works $[25,26]$, the last two properties are useful bona fide indicators of symmetry-protected topological orders.

(a) Spin profile. One characteristic feature of a $S=1$ Haldane chain with open boundary conditions is the presence of topologically protected fractionalized spin- $1 / 2$ end-states, a consequence of the broken $Z_{2} \times Z_{2}$ symmetry of the ground state [24]. These states can be represented as: $\left|\uparrow_{L}\right\rangle \otimes\left|\uparrow_{R}\right\rangle,\left|\uparrow_{L}\right\rangle \otimes\left|\downarrow_{R}\right\rangle,\left|\downarrow_{L}\right\rangle \otimes\left|\uparrow_{R}\right\rangle,\left|\downarrow_{L}\right\rangle \otimes\left|\downarrow_{R}\right\rangle$, and correspond to the fourfold-degenerate ground state in the thermodynamic limit $L \rightarrow \infty$. In order to detect these fractionalized spin excitations in our system, we have defined the spin profile as $\left\langle T_{j}^{z}\right\rangle=\left\langle\psi_{g}^{M^{z}=1}\left|T_{j}^{z}\right| \psi_{g}^{M^{z}=1}\right\rangle$, where $T_{j}^{z}$ is the $z$-projection of the total spin in the $j$-th rung $\mathbf{T}_{j}=\mathbf{S}_{j}+\mathbf{s}_{j}$, and $\left|\psi_{g}^{M^{z}=1}\right\rangle$ is the ground state of the system with total spin $M^{z}=1$ (where the visualization of the spin states at the ends is easier). In Fig. 2 we show $\left\langle T_{j}^{z}\right\rangle$ vs $j$ for different values of $J_{K}^{s} / J_{K}^{p}$ and for $L=80$. The presence of spin states localized at the edges can be clearly seen for $J_{K}^{s} / J_{K}^{p}=0$ and $J_{K}^{s} / J_{K}^{p}=0.85$, where 
PAPers in Physics, vol. 8, ART. 080005 (2016) / F. T. Lisandrini et al.

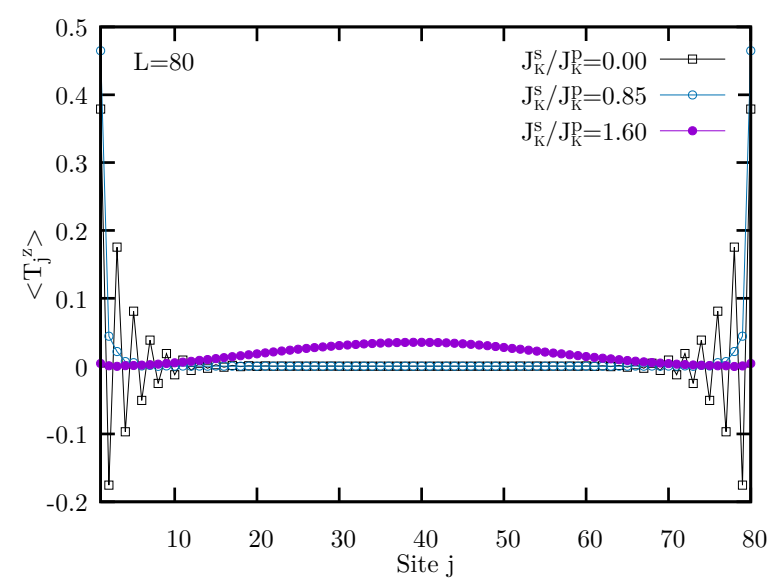

Figure 2: Spatial profile of $\left\langle T_{j}^{z}\right\rangle=$ $\left\langle\psi_{g}^{M^{z}=1}\left|T_{j}^{z}\right| \psi_{g}^{M^{z}=1}\right\rangle$, i.e., the $z$ component of the total spin in the supersite $j$, computed with the ground state of the subspace with total $M^{z}=1$ for $L=80$, and for parameters $J_{H} / t=1$ and $J_{K}^{p} / t=2$. The destruction of the topologically protected spin- $1 / 2$ states at the ends of the chain can be clearly seen as $J_{K}^{s} / J_{K}^{p}$ is increased from $J_{K}^{s} / J_{K}^{p}=0$ to $J_{K}^{s} / J_{K}^{p}=1.6$.

the spin density is accumulated at the ends of the system. Note that since we are working in the subspace $M^{z}=1$, this ground state corresponds to the state $\left|\uparrow_{L}\right\rangle \otimes\left|\uparrow_{R}\right\rangle$. For $J_{K}^{s} / J_{K}^{p}=1.6$, the magnetic edge-states have already disappeared, indicating that the onset of the topologically trivial phase must occur at lower values of $J_{K}^{s} / J_{K}^{p}$. However, while this analysis is useful to understand the nature of the topologically-ordered ground state, it does not allow a precise determination of the TQPT. To that end, we have studied the entanglement entropy and entanglement spectrum (see below).

(b) Entanglement entropy. We have also calculated the entanglement entropy (i.e., the von Neumann entropy of the reduced density matrix), defined as [49]

$$
S(L / 2)=-\operatorname{Tr} \hat{\rho}_{L / 2} \ln \hat{\rho}_{L / 2}=-\sum_{j} \Lambda_{j} \ln \Lambda_{j},
$$

where $\hat{\rho}_{L / 2}$ is the reduced density matrix obtained after tracing out half of the chain, and $\Lambda_{j}$ the corresponding eigenvalues of $\hat{\rho}_{L / 2}$, which are the squares of the Schmidt values. Recently, it has been clarified that the entanglement of a single quantum state is a crucial property not only from the perspective of quantum information, but also for condensed matter physics. In particular, the entanglement entropy has been shown to contain the quantum dimension, a property of topologicallyordered phases [50,51]. Hirano and Hatsugai [52] have computed the entanglement entropy of the open-boundary spin-1 Haldane chain and obtained the lower-bound value $S(L / 2)=\ln (4)=2 \ln (2)$ which, according to the edge-state picture in the thermodynamical limit $L \rightarrow \infty$, corresponds to the aforementioned 4 spin- $1 / 2$ edge states. In Fig. 3 we show the entanglement entropy of the system as a function of $J_{K}^{s} / J_{K}^{p}$, for different system sizes and in the subspace $M^{z}=0$, where we expect to find the ground state (i.e., the ground state of an even-numbered antiferromagnetic chain is a global singlet [53]). Near the critical region, the entanglement entropy grows due to the contribution of the bulk, and exactly at the TQPT the entanglement entropy is predicted to show a logarithmic divergence $S(L / 2) \sim \ln (L)$, characteristic of critical onedimensional systems [49]. As the size of the system is increased, the logarithmic divergence becomes narrower and its position shifts to larger values. Using the fitting function $J_{K, c}^{s}(L)=J_{K, c}^{s}(\infty)+a / L^{2}$, we have obtained the extrapolated critical point $J_{K, c}^{s}(\infty) \approx 1.11 J_{K}^{p}$ in the thermodynamic limit, see inset (a) in Fig. 3. Note that this value is smaller than the predicted value $J_{K, c}^{s}=2 J_{K}^{p}$ using the field-theory analysis of the previous section. We believe this to be the effect of the neglected marginal or irrelevant operators, which renormalize non-universal quantities such as the critical point.

We have also confirmed the logarithmic scaling of the entanglement entropy at the critical point, i.e., $S_{\max }(L / 2)=\alpha \ln (L)+$ constant, and we have obtained a prefactor $\alpha=0.052$, see inset (b) in Fig. 3. A detailed analysis of this value and its connection to the corresponding central charge value of the conformal field theory is beyond the scope of the present work and is left to a subsequent publication.

In Fig. 3, note that for $J_{K}^{s} / J_{K}^{p}<J_{K, c}^{s} / J_{K}^{p}$, the value of the entanglement entropy roughly corresponds to $S(L / 2) \sim 2 \ln (2)$, consistent with the theoretical predictions in the Haldane phase. Values of $S(L / 2)$ which are below the predicted lower- 


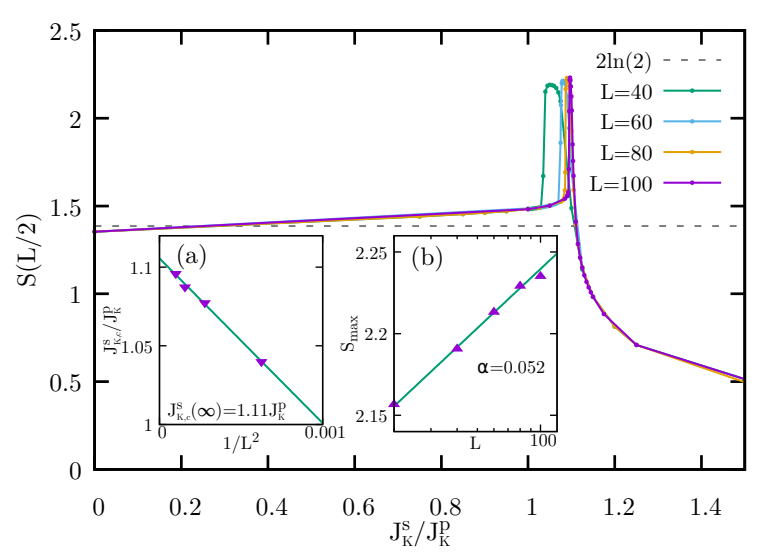

Figure 3: Entanglement entropy of the reduced density matrix, $S(L / 2)$, as function of $J_{K}^{s} / J_{K}^{p}$ for different lattice sizes. The entropy is computed within the subspace $M^{z}=0$ and for parameters $J_{H} / t=1$ and $J_{K}^{p} / t=2$. The maximum of $S(L / 2)$ indicates the position of the critical point. Inset (a): Finite-size scaling of the critical point, i.e., $J_{K, c}^{s}(L)=J_{K, c}^{s}(\infty)+a / L^{2}$, from where the value in the thermodynamic limit $J_{K, c}^{s}(\infty) \approx 1.11 J_{K}^{p}$ is obtained. Inset (b): Maximum entropy $S_{\max }(L / 2)$ vs $L$. The results reproduce the predicted logarithmic divergence $S_{\max }(L / 2)=\alpha \ln (L)+$ constant, with the fitting constant $\alpha=0.052$.

bound are presumably due to finite-size effects, which result in a decrease of the effective quantum dimension in small systems [52]. For $J_{K}^{s} / J_{K}^{p}>$ $J_{K, c}^{s} / J_{K}^{p}, S(L / 2)$ tends to zero as expected for a topologically trivial ground state. This result can be easily understood in the limit $J_{K}^{s} / J_{K}^{p} \rightarrow \infty$, where we expect the ground state to factorize as a product of local singlets (we recall that we are working in the subspace $M^{z}=0$ ), for which $S(L / 2)=0$.

(c) Degeneracy of the entanglement spectrum Finally, we focus on the full entanglement spectrum of the reduced density-matrix. Degeneracies in the entanglement spectrum are intimately related to the existence of discrete symmetries which protect the topological order. In particular, as shown in Ref. [25], an even degeneracy constitutes the most distinctive feature of the Haldane phase. This fact allows to make an interesting connection

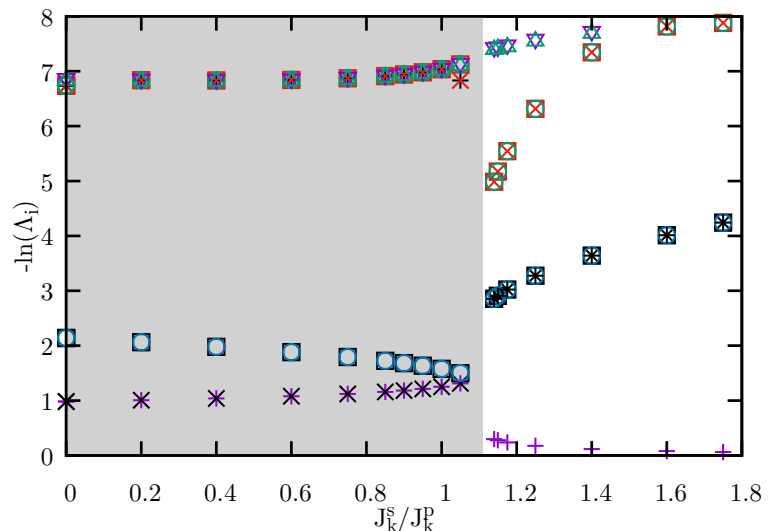

Figure 4: The largest eigenvalues of the entanglement spectrum (extrapolated to the thermodynamic limit $L \rightarrow \infty)$, as a function of $J_{K}^{s}$. The results were obtained in the subspace $M^{z}=0$. The gray zone corresponds to the Haldane phase, where the degeneracy in the spectrum of eigenvalues is even.

between the theory of symmetry-protected topological phases and the theory of topological Kondo insulators in one dimension, and might have important implications in the understanding of strongly interacting topological phases of fermions. In Fig. 4 we show the evolution of the largest eigenvalues extrapolated to the thermodynamic limit of the density matrix as a function of the parameter $J_{K}^{s} / J_{K}^{p}$, obtained in the subspace $M^{z}=0$. Note that for $J_{K}^{s} / J_{K}^{p}<J_{K, c}^{s} / J_{K}^{p} \sim 1.11$ (i.e., gray-shaded area), the degeneracy of the eigenvalues is even, as expected for the Haldane phase. In contrast, for values $J_{K}^{s} / J_{K}^{p}>J_{K, c}^{s} / J_{K}^{p}$, the evendegeneracy breaks down, indicating the onset of the trivial phase. In particular, note the evolution of the largest eigenvalue of the density matrix $\Lambda_{0} \approx 1$ (i.e., lowest "+" symbol) which becomes non-degenerate.

\section{Conclusions}

Using a combination of techniques, i.e., a fieldtheoretical analysis and the density-matrix renormalization group (DMRG), an essentially exact method in one dimension, we have studied the tran- 
PAPers in Physics, vol. 8, ART. 080005 (2016) / F. T. Lisandrini et al.

sition from a topological to a non-topological phase in a model for a one-dimensional strongly interacting topological Kondo insulator. As a prototypical topological quantum phase transition with no Landau-type local order-parameter, one must resort to global quantities characterizing the ground state. In this work, we have shown that the entanglement entropy and the entanglement spectrum can be used to characterize a topological Kondo insulator in one dimension. This system was originally understood and classified according to noninteracting topological invariants (i.e., Chern numbers), employing approximate large- $N$ mean-field methods [20]. Here, by the means of the DMRG, we have shown that a more appropriate way to understand this system is by using the concepts developed for symmetry-protected topological phases [25-28]. In particular, for parameters $J_{H} / t=1$ and $J_{K}^{p} / t=2$, we have obtained the value of the critical point $J_{K, c}^{s} / J_{K}^{p} \simeq 1.11$ in the thermodynamic limit $L \rightarrow \infty$. This value is smaller than the expected from the qualitative field-theoretical estimation $J_{K, c}^{s} / J_{K}^{p}=2$, a fact that is presumably originated in the effect of marginal or irrelevant operators, which were neglected in the qualitative analysis and which renormalize a non-universal quantity such as the critical point.

Acknowledgements - F.T.L, A.O.D. and C.J.G. acknowledge support from CONICET-PIP 11220120100389CO. A.M.L acknowledges support from PICT-2015-0217 of ANPCyT.

[1] B A Bernevig, T L Hughes, S C Zhang, Quantum spin hall effect and topological phase transition in $\mathrm{HgTe}$ quantum wells, Science 314, 1757 (2006).

[2] L Fu, C L Kane, Topological insulators with inversion symmetry, Phys. Rev. B 76, 045302 (2007).

[3] H Zhang, C X Liu, X L Dai, Z Fang, S C Zhang, Topological insulators in Bi2Se3, Bi2Te3 and Sb2Te3 with a single Dirac cone on the surface, Nat. Phys. 5, 438 (2009).

[4] M König, S Wiedmann, C Brüne, A Roth, H Buhmann, L W Molenkamp, X L Qi, S C
Zhang, Quantum spin hall insulator state in HgTe quantum wells, Science 318, 766 (2007).

[5] D Hsieh, D Qian, L Wray, Y Xia, Y S Hor, R J Cava, M Z Hasan, A topological dirac insulator in a quantum spin hall phase, Nature 452, 970 (2008).

[6] Y Xia, D Qian, L Hsieh, D Wray, A Pal, H Lin, A Bansil, D Grauer, Y S Hor, R J Cava, M Z Hasan, Observation of a large-gap topologicalinsulator class with a single dirac cone on the surface, Nat. Phys. 5, 398 (2009).

[7] A Altland, M R Zirnbauer, Nonstandard symmetry classes in mesoscopic normalsuperconducting hybrid structures, Phys. Rev. B 55, 1142 (1997).

[8] A Kitaev, Periodic table for topological insulators and superconductors, AIP Conf. Proc. 1134, 22 (2009).

[9] S Ryu, A P Schnyder, A Furusaki, A W W Ludwig, Topological insulators and superconductors: tenfold way and dimensional hierarchy, New J. Phys. 12, 065010 (2010).

[10] M Dzero, K Sun, V Galitski, P Coleman, Topological Kondo insulators, Phys. Rev. Lett. 104, 106408 (2010).

[11] M Dzero, K Sun, P Coleman, V Galitski, Theory of topological Kondo insulators, Phys. Rev. B 85, 045130 (2012).

[12] M Dzero, J Xia, V Galitski, P Coleman, Topological Kondo Insulators, Annu. Rev. Condens. Matter Phys. 7, 249 (2016).

[13] N Read, D M Newns, On the solution of the Coqblin-Schreiffer Hamiltonian by the large$n$ expansion technique, J. Phys. C 16, 3273 (1983).

[14] P Coleman, Mixed valence as an almost broken symmetry, Phys. Rev. B 35, 5072 (1987).

[15] D M Newns, N Read, Mean-field theory of intermediate valence/heavy fermion systems, Adv. Phys. 36, 799 (1987). 
PAPers in Physics, vol. 8, ART. 080005 (2016) / F. T. Lisandrini et al.

[16] S Wolgast, C Kurdak, K Sun, J W Allen, D J Kim, Z Fisk, Low-temperature surface conduction in the Kondo insulator $\mathrm{SmB}_{6}$, Phys. Rev. B 88, 180405 (2013).

[17] X Zhang, N P Butch, P Syers, S Ziemak, R L Greene, J Paglione. Hybridization, inter-ion correlation, and surface states in the Kondo insulator $\mathrm{SmB}_{6}$, Phys. Rev. X 3, 011011 (2013).

[18] N Xu et al., Direct observation of the spin texture in $\mathrm{SmB}_{6}$ as evidence of the topological Kondo insulator, Nat. Commun. 5, 4566 (2014).

[19] D J Kim, J Xia, Z Fisk, Topological surface state in the Kondo insulator samarium hexaboride, Nat. Mater. 13, 466 (2014).

[20] V Alexandrov, P Coleman, End states in a one-dimensional topological Kondo insulator in large-n limit, Phys. Rev. B 90, 115147 (2014).

[21] A M Lobos, A O Dobry, V Galitski, Magnetic end states in a strongly interacting one-dimensional topological Kondo insulator, Phys. Rev. X 5, 021017 (2015).

[22] I Affleck, T Kennedy, E H Lieb, H Tasaki, Rigorous results on valence-bond ground states in antiferromagnets, Phys. Rev. Lett. 59, 799 (1987).

[23] I Affleck, T Kennedy, E H Lieb, H Tasaki, Valence bond ground states in isotropic quantum antiferromagnets, Commun. Math. Phys. 115, 477 (1988).

[24] T Kennedy, H Tasaki, Hidden $z_{2} \times z_{2}$ symmetry breaking in haldane gap antiferromagnets, Phys. Rev. B 45, 304 (1992).

[25] F Pollmann, A M Turner, E Berg, M Oshikawa, Entanglement spectrum of a topological phase in one dimension, Phys. Rev. B 81, 064439 (2010).

[26] F Pollmann, E Berg, A M Turner, M Oshikawa, Symmetry protection of topological phases in one-dimensional quantum spin systems, Phys. Rev. B 85, 075125 (2012).
[27] Z C Gu, X G Wen, Tensor-entanglementfiltering renormalization approach and symmetry-protected topological order, Phys. Rev. B 80, 155131 (2009).

[28] X Chen, Z C Gu, X G Wen, Classification of gapped symmetric phases in one-dimensional spin systems, Phys. Rev. B 83, 035107 (2011).

[29] A Mezio, A M Lobos, A O Dobry, C J Gazza, Haldane phase in one-dimensional topological Kondo insulators, Phys. Rev. B 92, 205128 (2015).

[30] I Hagymási, Ö Legeza, Characterization of a correlated topological Kondo insulator in one dimension, Phys. Rev. B 93, 165104 (2016).

[31] O Zachar, S A Kivelson, V J Emery, Exact results for a $1 D$ Kondo lattice from bosonization, Phys. Rev. Lett. 77, 1342 (1996).

[32] A E Sikkema, I Affleck, S R White, Spin gap in a doped Kondo chain, Phys. Rev. Lett. 79, 929 (1997).

[33] O Zachar, A M Tsvelik, One dimensional electron gas interacting with a Heisenberg spin-1/2 chain. Phys. Rev. B 64, 033103 (2001).

[34] O Zachar, Staggered liquid phases of the onedimensional Kondo-Heisenberg lattice model, Phys. Rev. B 63, 205104 (2001).

[35] E Berg, E Fradkin, S A Kivelson, Pair-densitywave correlations in the Kondo-Heisenberg model, Phys. Rev. Lett. 105, 146403 (2010).

[36] A Dobry, A Jaefari, E Fradkin, Inhomogeneous superconducting phases in the frustrated Kondo-Heisenberg chain, Phys. Rev. B 87, 245102 (2013).

[37] G Y Cho, R Soto-Garrido, E Fradkin, Topological Pair-Density-Wave Superconducting States, Phys. Rev. Lett., 113, 256405 (2014).

[38] D G Shelton, A A Nersesyan, A M Tsvelik, Antiferromagnetic spin ladders: Crossover between spin $S=1 / 2$ and $S=1$ chains, Phys. Rev. B 53, 8521 (1996). 
Papers in Physics, VOL. 8, ART. 080005 (2016) / F. T. Lisandrini et al.

[39] A O Gogolin, A A Nersesyan, A M Tsvelik, $\mathrm{L} \mathrm{Yu,} \mathrm{Zero-modes} \mathrm{and} \mathrm{thermodynamics} \mathrm{of} \mathrm{dis-}$ ordered spin-1/2 ladders, Nucl. Phys. B 540, 705 (1999).

[40] P Lecheminant, E Orignac, Magnetization and dimerization profiles of the cut two-leg spin ladder and spin-1 chain, Phys. Rev. B 65, 174406 (2002).

[41] N J Robinson, F H L Essler, E Jeckelmann, A M Tsvelik, Finite wave vector pairing in doped two-leg ladders, Phys. Rev. B, 85, 195103 (2012).

[42] V J Emery, Theory of the one-dimensional electron gas, In: Highly conducting onedimensional solids, Eds. J T Devreese, R P Evrard, V E van Doren, Pag. 247, Plenum, New York (1979).

[43] J Sólyom, The fermi gas model of onedimensional conductors, Adv. in Phys. 28, 201 (1979).

[44] J von Delft, H Schoeller, Bosonization for beginners refermionization for experts, Ann. Phys. (N. Y.) 7, 225 (1998).

[45] T Giamarchi, Quantum Physics in One Dimension, Oxford University Press, Oxford (2004).
[46] A O Gogolin, A A Nersesyan, A M Tsvelik, Bosonization and Strongly Correlated Systems, Cambridge University Press, Cambridge (1999).

[47] I Affleck, In E. Brezin and J. Zinn-Justin, editors, Fields, Strings and Critical Phenomena, pag. 563, Elsevier Science, Amsterdam (1988).

[48] A A Nersesyan, A O Gogolin, Fabian H L Essler, Incommensurate spin correlations in Heisenberg spin-1/2 zig-zag ladders, Phys. Rev. Lett. 81, 910 (1998).

[49] U Schollwöck, The density-matrix renormalization group, Rev. Mod. Phys. 77, 259 (2005).

[50] A Kitaev, J Preskill, Topological Entanglement Entropy, Phys. Rev. Lett. 96, 110404 (2006).

[51] M Levin, X G Wen, Detecting Topological Order in a Ground State Wave Function, Phys. Rev. Lett. 96, 110405 (2006).

[52] T Hirano, Y Hatsugai, Entanglement Entropy of One-dimensional Gapped Spin Chains, J. Phys. Soc. Japan 76, 074603 (2007).

[53] A Auerbach, Interacting Electrons and Quantum Magnetism, Springer, Berlin (1998). 